\title{
Combining Genetic Algorithm and Switches for Improving the Quality of Compression
}

\author{
Digvijay Singh Thakur ${ }^{1}$, Prabhakar Sharma ${ }^{2}$ \\ M.Tech Scholar, Department of Computer Science \& Engineering, Raipur Institute of Technology, Raipur C.G. ${ }^{1}$ \\ Assistant Professor, Department of Computer Science \& Engineering, Raipur Institute of Technology, Raipur C.G. ${ }^{2}$
}

\begin{abstract}
Genetic algorithm and neural system based strategies are intense technique for taking care of genuine issues and has been generally considered for its applications in territories of picture pressure. For picture compression.ga based learning for neural system is experiencing condemnation of moderate union and low quality of pressure. Neural systems for tuning more often than not have a settled structure. The quantity of associations must be sufficiently huge to fit a given application. This may cause the neural system structure to be pointlessly intricate and increment the usage cost. The present work proposes a neural system with switches. By doing this, the proposed neural system can learn both the information yield connections of an application and the system structure. The trial results demonstrate that the method performed productively then the calculation variant without switches
\end{abstract}

Keywords: Image Processing, Image Compression, Genetic Algorithm, Switches.

\section{INTRODUCTION}

Picture preparing is a system to change over a picture into computerized frame and play out a few activities to get an improved picture or to separate some accommodating data from it. It is a sort of flag regulation which takes an info picture, similar to video edge or photo and yield is generally a picture or attributes related with that picture. A picture is simply a two-dimensional flag which is characterized by the numerical capacity $f(x, y)$ where, $x$ and $y$ are the two coordinates horizontally and vertically and the amplitude of $\mathrm{f}$ at any pair of coordinate $(\mathrm{x}, \mathrm{y})$ is called the intensity or gray level of the image at that point. The $(\mathrm{x}, \mathrm{y})$ and amplitude values of $\mathrm{f}$ are all finite discrete quantities. Usually, Image Processing framework incorporates regarding pictures as two-dimensional signs while applying predefined calculations so as to remove data or perform reclamation. Picture handling essentially incorporates the accompanying three stages:

- $\quad$ Importing the picture with optical scanner or by advanced photography

- $\quad$ Analysing and picture control which incorporates information pressure and picture improvement and spotting designs from pictures like satellite photos.

- e Output is the last stage in which result can be changed picture or report that depends on picture examination.. Image compression coding is to store the picture into bit-stream as minimal as would be prudent and to show the decoded picture in the screen as correct as could reasonably be expected. At the point when the encoder gets the first picture document, the picture record will be changed over into a progression of parallel information, which is known as the bit-stream. The decoder at that point gets the encoded bit-stream and interprets it to frame the decoded picture. In the event that the aggregate information amount of the bit-stream is not as much as the aggregate information amount of the first picture, at that point this is called picture pressure. The pressure proportion is characterized as takes after:

$$
\mathrm{Cr}=\frac{\mathrm{n} 1}{\mathrm{n} 2}
$$

Where $\mathrm{n} 1$ is the data rate of original image and $\mathrm{n} 2$ is that of the encoded bit-stream.

With a specific end goal to assess the execution of the picture pressure coding, it is important to characterize an estimation that can appraise the contrast between the first picture and the decoded picture. Two basic utilized estimations are the Mean Square Error (MSE) and the Peak Signal to Noise Ratio (PSNR) individually. $\mathrm{f}(\mathrm{x}, \mathrm{y})$ is the pixel estimation of the first picture, and $f^{\prime}(x, y)$ is the pixel estimation of the decoded picture. Most picture pressure frameworks are intended to limit the MSE and boost the PSNR. The conditions for discovering MSE and PSNR are as per the following:

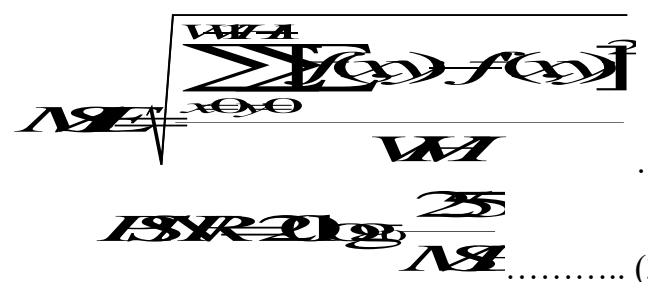




\section{ANN AND GENETIC ALGORITHM BASED IMAGE COMPRESSION}

An Artificial Neural Network (ANN) is a data preparing worldview that is motivated by the way organic sensory systems, for example, the mind, process data. The key component of this worldview is the novel structure of the data handling framework. It is made out of countless interconnected preparing components (neurons) working as one to tackle particular issues. ANNs, similar to individuals, learn by illustration. An ANN is arranged for a particular application, for example, design acknowledgment or information characterization, through a learning procedure. Learning in natural frameworks includes acclimations to the synaptic associations that exist between the neurons. This is valid for ANNs also. Neural systems, with their momentous capacity to get importance from muddled or loose information, can be utilized to separate examples and distinguish patterns that are too perplexing to possibly be seen by either people or other PC methods. A prepared neural system can be thought of as a "specialist" in the classification of data it has been given to break down. This master would then be able to be utilized to give projections given new circumstances of intrigue and reply "imagine a scenario in which" questions:

1. Adaptive learning: A capacity to figure out how to do undertakings in view of the information given for preparing or introductory experience.

2. Self-Organization: An ANN can make its own association or portrayal of the data it gets amid learning time.

3. Real Time Operation: ANN calculations might be done in parallel, and exceptional equipment gadgets are being composed and produced which exploit this ability.

4. Fault Tolerance via Redundant Information Coding: Partial obliteration of a system prompts the relating debasement of execution. In any case, some system capacities might be held even with significant system harm.

Genetic algorithms (GAs) in light of the mechanics of normal determination and characteristic hereditary qualities. Hereditary calculations (GAs) were designed by John Holland in the 1960s and were produced by Holland and his understudies and partners at the University of Michigan in the 1960s and the 1970s. GA depends on moving from one populace of "chromosomes" (e.g., series of zeros, or "bits") to another populace by utilizing a sort of "normal determination" together with the genetics-inspired administrators of hybrid, change, and reversal. Every chromosome comprises of "qualities" (e.g., bits). The determination administrator picks those chromosomes in the populace that will be permitted to recreate, and by and large the fitter chromosomes deliver more posterity than the less fit ones. Hybrid trades subparts of at least two chromosomes. Transformation haphazardly changes the allele estimations of a few areas in the chromosome. In GAs, every person in the inquiry space is coded as a chromosome, which comprises of the characters (qualities) 1's and 0 's. So first we pick haphazardly an arrangement of chromosomes to constitute an underlying populace. At that point, every chromosome is assessed in light of a wellness work that characterizes optimality. From that point forward, an arrangement of fundamental activities is connected to the number of inhabitants in the chromosomes. Determination is a task which chooses the chromosomes as per their wellness esteems. Thus, if a chromosome has higher wellness esteem, it will have a high opportunity to deliver offsprings into the people to come. In the hybrid activity, the chose guardians are crossed to make new chromosomes by swapping qualities between guardians. Transformation is the last task which modifies the quality of a few chromosomes (as a rule with low likelihood). The GA stages are rehashed until the point that a streamlining arrangement is found or an end paradigm is satisfied. For picture pressure the calculation is as per the following:

1. Chromosomes are introduced where every chromosome speak to a codebook . The mark $(\mathrm{I}=1,2,3, \ldots \ldots \ldots, \mathrm{M})$ of the preparation vector is seen as a quality, hence the essential individual is comprised of $\mathrm{M}$ qualities which can part into $\mathrm{N}$ units, every unit is comprised of a few names which have a place with this unit. $\mathrm{N}$ code words are the middle vector of the preparation vectors in every unit. Each square in picture references its code word utilizing 8-bits. The codebook will be a variety of two measurements and length of code word is 256 , while the quantity of segments in the codebook will be changes as per the info picture estimate. Every unit in codebook is grouped utilizing squares of picture utilizing the squared mistake contortion utilizing the accompanying recipe.

$$
\mathrm{d}\left(\mathrm{X}, \mathrm{Y}_{\mathrm{j}}\right)=\sum_{\mathrm{l}=1}^{\mathrm{k}}\left(\mathrm{x}_{\mathrm{l}}-\mathrm{y}_{\mathrm{jl}}\right) \ldots
$$

Where $\mathrm{X}$ represent the block of training image $\mathrm{Y}$ represents the unit in the codebook. According to the above step the generations are initialized.

2. The chromosome with best wellness will be picked as best agent chromosome for think about picture. For every person, the projects figure the general contortion as indicated by the accompanying equation.

$$
\mathrm{D}=\sum_{\mathrm{r}=1}^{\mathrm{N}} \sum_{\mathrm{i}=1}^{\mathrm{n}_{\mathrm{lr}}^{(\mathrm{t})}}\left(\mathrm{X}_{\mathrm{a}_{\mathrm{li}}^{(\mathrm{t}) \mathrm{r}}}-\mathrm{Y}_{\mathrm{r}}^{(\mathrm{t})}\right)^{2}
$$

Fitness function for each chromosome calculated according to the formula

$$
\text { fitness }=\frac{1}{\mathrm{D}} \text {. }
$$


3. Choice process is performed between two arbitrarily picked guardians utilizing Tournament Selection technique. On the off chance that the wellness estimation of the primary parent is equivalent to the wellness estimation of the second parent, one of them will be chosen arbitrarily.

4. Hybrid process is performed utilizing two point hybrids by trading the qualities between these focuses in each parent to deliver two new posterity. This is performed with a specific likelihood; generally the two guardians are replicated as posterity.

5. In subsequent stage a quality is haphazardly chosen from every person and the focal point of that quality is supplanted with intend to influence this pixel to take esteem closest to its neighbor pixels.

6. The end criteria are taken as no of ages and mistake esteems. The cycle ends when one of the condition is fulfilled.

\section{III.RELATED WORK}

The work in [1] researches the execution of ANN utilizing GA in the utilization of picture pressure for getting ideal arrangement of weights. Coordinate strategy for pressure has been actualized with neural system to get the added substance advantage for security of compacted information. The tests uncover that the typical BP with appropriate parameters convey great sum up limit with respect to pressure and is considerably quicker contrasted with before work in the writing, in light of combined circulation work. Further, the outcomes acquired show that general thought regarding GA, it plays better finished inclination average based learning, isn't appropriate for picture pressure.

Another work [2] presented a pristine picture pressure component in light of abusing the connection between fragmentary numbers and their comparing remainder portrayal. Each sub-picture is mapped to a partial number in view of the RGB portrayal, and afterward diminished to a proficient remainder.

In [3] the interest of utilizing hereditary calculations is clarified by the huge hunt to locate a nearby division that is diminished to brief remainder. The strategy demonstrated an impressive pressure proportion when the slightest noteworthy bits of every byte are changed, consequently, the picture quality is safeguarded while accomplishing high pressure proportion. Bringing down the inquiry intricacy of fitting between cluster square and space name obstruct from fractal picture pressure is among the most dynamic research regions of late.

Work in [4] advances an enhanced hereditary calculation recommended for getting coordinating space squares to ensure against the early union of GA into a problematic individual, to make singular assorted variety and to enhance GA's pursuit effectiveness. For each range obstruct, an enhanced GA's methodology is to encode the place of chasing space square utilizing Gray code, indicate readiness for least separation of current range square coordinating with chasing area square, select ideal individual security plan and adjust GA's controlled parameters of hybrid and transformation likelihood adaptively.

As indicated by [5] while the switch-based system is among the most essential and solid instruments recommended in the M-NN, an expansion of this rule may make its impact considerably more noteworthy. Rather than simply taking into account the evacuation of associations, switches are set on the concealed hubs inside the system, in this manner taking into consideration the pruning of the availability and size. Clients never again need to prepare and look at the system for different quantities of hubs, even as one go through using a genuinely high number of shrouded hubs will allow the calculation to kill some superfluous hubs and locate the ideal size individually]

\section{IV.METHODOLOGY}

A. Problem Statement: GA based learning for neural system is experiencing condemnation of moderate merging and low quality of pressure. Neural systems for tuning for the most part have a settled structure. The quantity of associations must be sufficiently extensive to fit a given application. This may cause the neural system structure to be pointlessly mind boggling and increment the execution cost. A portion of the issues in current pressure systems are:

- JPEG utilizes some lossless calculations, yet in addition utilizes quantization. The quantization of the record can result in lost information. The measure of quantization is variable. JPEG can lessen document sizes 5:1 with negligible debasement and upwards to 20:1 with noteworthy corruption. The JPEG calculation starts by part the picture into three separate channels making three separate pictures. Each shading channel picture is broken into fragments that are 8 pixels by 8 pixels in estimate ( $8 \times 8$ squares). Each $8 \times 8$ square is spoken to by a numerical capacity making another $8 \times 8$ square. Quantization is connected in view of the "quality" level the client chooses. The more quantization connected the littler the record measure bringing about more prominent misfortune. JPEG can be lossless if the quantization level is set to zero. After quantization, the $8 \times 8$ squares are reassembled and the packed shading channels are consolidated once more into one picture. 
- $\quad$ JPEG2000 is like JPEG yet utilizes an alternate numerical capacity. It doesn't fragment the picture utilizing 8x8 squares as JPEG does. It utilizes descending insertion to make littler adaptations of the picture and applies a scientific capacity taken after by quantization to accomplish pressure. Contrasted with standard JPEG, JPEG2000 can accomplish a more noteworthy pressure of picture documents while keeping up a similar picture quality. JPEG2000 can decrease record sizes up to 20:1 with insignificant debasement. It can pack up to 80:1; nonetheless, huge debasement happens at this level.

- When thinking about pressure, organizations need to adjust cost, work process, time, and picture quality. Pressure can make investigation more troublesome despite the fact that the picture is as yet usable.

In the present work a neural system with changes acquainted with its connections is proposed. By doing this, the proposed neural system can learn both the info yield connections of an application and the system structure. The quantity of concealed hubs ought to be picked physically beginning from a modest number. The quantity of concealed hubs ought to be expanded if the learning execution as far as wellness esteem isn't adequate. Utilizing the enhanced $\mathrm{GA}$, the structure and the parameters of the neural system can be tuned.

\section{B. Proposed Algorithm}

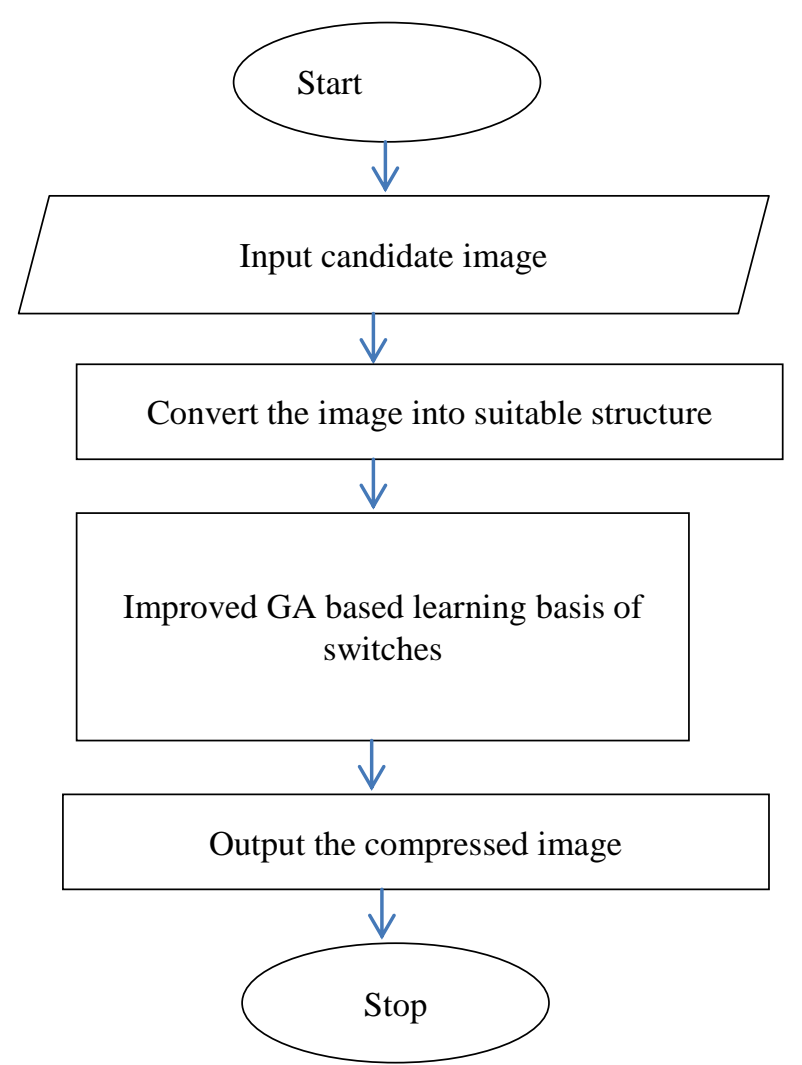

Figure 1: Improved GA based technique for image compression

The overall image compression is carried out using Neural Network. In order to adjust the weights of the network GA based switches are used. The switches are binary functions that lie on the connections between nodes. In other words, if some parameter is greater than or equal to 0 , the switch is turned on and a connection exists between the two nodes, but if is less than 0 , the switch is turned o and the connection does not exist. The equation can be represented as:

$$
f(x)=\left\{\begin{array}{l}
0 \text { if } x<0 \\
1 \text { if } x>0
\end{array}, x \in R\right.
$$

Instead of only allowing for the removal of connections, switches have been placed on the hidden nodes within the network, thus allowing for the tuning of both connectivity and size. Users no longer have to train and test the network for various numbers of nodes, as one run-through using a rather large number of hidden nodes will allow the algorithm to turn off any unnecessary nodes and the optimal size on its own. The node switches behave in the same manner as those found on the connections. A parameter will be tuned using the GA, and will then be compared to a threshold value of 0 . If the parameter is greater than or equal to the threshold, the node is turned on; if not, it is switched off. 


\section{Step 1:}

1. Initialize population on the basis of training samples.

2. Evaluate fitness of the initial population.

3. Apply tournament selection operation.

4. Apply mutation operation.

5. Output best weights.

\section{Step 2:}

1. Input pixel values of candidate image and split into small sub images.

2. Image compression is completed by the quantum state from the input layer to hidden layer.

3. Use GA method based on mean square error to adjust the weight, until the image reconstruction error less than target error.

The hidden layer output is the result of image compression.

\section{RESULTS AND DISCUSSION}

The experimental was simulated on Matlab platform and results were recorded on the basis of PSNR and MSE table which is as mentioned below:

Table 1: MSE comparison

\begin{tabular}{|ll|ll|ll|}
\hline $\begin{array}{l}1 . \\
\text { Name }\end{array}$ & Image & $\begin{array}{l}2 . \\
3 .\end{array}$ & $\begin{array}{l}\text { Existing } \\
\text { Value }\end{array}$ & $\begin{array}{l}\text { V. } \\
\text { Value }\end{array}$ & Proposed \\
\hline 5. & Lenna.jpg & 6. & 77 & 7. & 27 \\
\hline 8. & Camera.jpg & 9. & 52 & 10. & 13 \\
\hline 11. & People.jpg & 12. & 20 & 13. & 10.5 \\
\hline 14. & Fruits.jpg & 15. & 39 & 16. & 11 \\
\hline 17. & Flowers.jpg & 18. & 75 & 19. & 15 \\
\hline
\end{tabular}

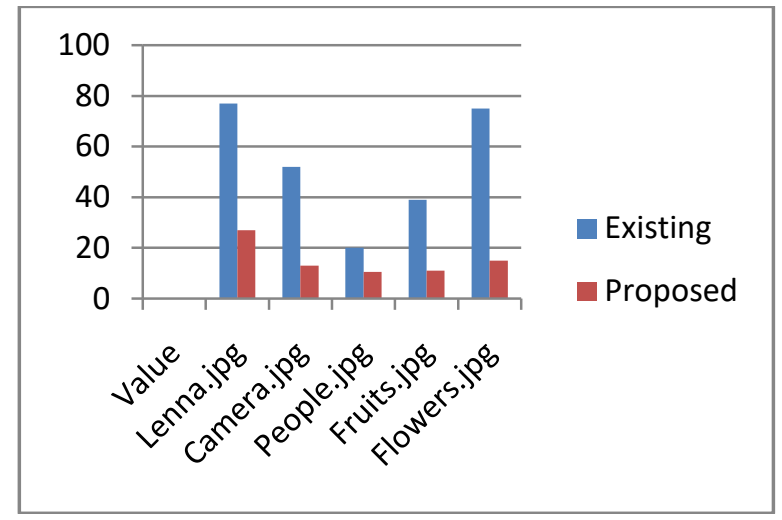

Figure 2: MSE comparison chart

Table 2: PSNR comparison

\begin{tabular}{|ll|ll|ll|}
\hline $\begin{array}{l}\text { 20. } \\
\text { Name }\end{array}$ & Image & $\begin{array}{l}21 . \\
22 .\end{array}$ & $\begin{array}{l}\text { Existing } \\
\text { Value }\end{array}$ & $\begin{array}{l}23 . \\
\text { Value }\end{array}$ & Proposed \\
\hline 24. & Lenna.jpg & 25. & 29.2659 & 26. & 27 \\
\hline 27. & Camera.jpg & 28. & 30.9708 & 29. & 25 \\
\hline 30. & People.jpg & 31. & 35.1205 & 32. & 28 \\
\hline 33. & Fruits.jpg & 34. & 32.2202 & 35. & 26 \\
\hline 36. & Flowers.jpg & 37. & 29.3802 & 38. & 26.444 \\
\hline
\end{tabular}


Vol. 7, Issue 8, August 2018

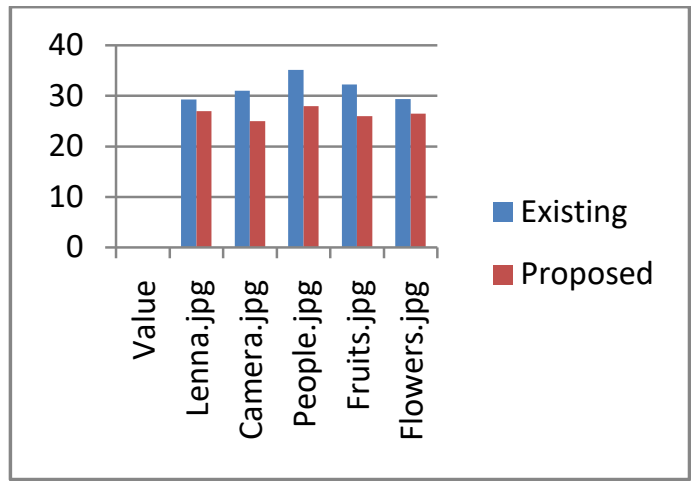

Figure 3: PSNR comparison chart

\section{CONCLUSION}

In this work, an improved switch based algorithm for image compression is proposed. In first step the Genetic algorithm is used to produce best weights for tuning parameters for adjusting weights in Neural Network that acts as switches to switch the layer on or off. Based on the switched the neural network is used for image compression and reconstructions. The proposed method gave a very good PSNR values and visual quality for the reconstructed grey level images. It produces a good compression ratio for grey level types of images. Future work includes developing a method that produces best weights for colour images.

\section{REFERENCES}

[1] Modeling of neural image compression using GA and BP: a comparative approach, G.G Rajput, Vrinda Shivashetty \& Manoj Kumar Singh, (IJACSA) International Journal of Advanced Computer Science and Applications, Special Issue on Image Processing and Analysis ,2012

[2] Image Compression Based on Genetic Algorithm Optimization, Mohammed Omari and Salah Yaichi, 2015 IEEE.

[3] L. Xi, L. Zhang. "A Study of Fractal Image Compression Based on an Improved Genetic Algorithm,” International Journal of Nonlinear Science, 2007, vol.3, no.2, pp. 116-124.

[4] A Modified Genetic Algorithm and Switch-Based Neural Network Model Applied to Misuse-Based Intrusion Detection, Ian Stewart, Queen's University ,2009.

[5] Fractal Image Compression Based on Multi-Objective Genetic Algorithm, Prashant Kashirao Adakane and Prof. Onkar Nath Thakur, International Journal Of Innovative Trends In Engineering (Ijite), 2015.

[6] A Genetic Algorithm Based K-Means Algorithm For Compressing Images, M.Mary Shanthi Rani, International Journal Of Engineering And Computer Science ISSN: 2319-7242 Volume 4 Issue 9 Sep 2015.

[7] Integrated Particle Swarm Optimization and Genetic Algorithm Based Compression for Reduction of Blocking Artifacts, Prabhjeet kaur, Er. Parminder Singh, (IJCSIT) International Journal of Computer Science and Information Technologies, Vol. 6 (5), 2015.

[8] H. S Soliman, M. Omari, "A neural networks approach to image data compression," Applied Soft Computing, 2006.

[9] S. Ramakrishnan, K. Rose, and A. Gersho, "Constrained-Storage Vector Quantization with a Universal Codebook," IEEE, 1998

[10] H. S. Soliman, M. Omari, "Image Compression using Neural Nets, Hybrid ART/Kohonen Neural Model for Document Image Compression," ANNIE 2002, University of Missouri-Rolla, MO, November 2002

[11] T. Kohonen, "Self-Organizing Maps, Optimization Approaches," Artificial Neural Networks, p 981-990, Elsevier Science Publishers B.V. (North-Holland) 1991.

[12] J. M. Zurada, "Introduction to Artificial Neural System", PWS Publishing Company, 1995.

[13] L. Xi, L. Zhang. "A Study of Fractal Image Compression Based on an Improved Genetic Algorithm," International Journal of Nonlinear Science, 2007, vol.3, no.2, pp. 116-124.

[14] M. Melanie, "An Introduction to Genetic Algorithms", a Bradford Book The MIT Press, 1999.

[15] Brahimi, Z. and Saadi, K.A. (2004) Color Image Coding based on Embedded Wavelet Zerotree and Scalar Quantization, ICPR, 17 ${ }^{\text {th }}$ International Conference on Pattern Recognition (ICPR'04), Vol. 1, Pp. 504-507. 